\title{
The successful endoscopic hemostasis factors in bleeding from advanced gastric cancer
}

\author{
Kang Hun Koh • Kang Kim • Dae Hun Kwon • Bum Su Chung • Ji Youn Sohn • \\ Dae Seon Ahn • Byung Jun Jeon · Seong Hun Kim • In Hee Kim • \\ Sang Wook Kim $\cdot$ Seung Ok Lee $\cdot$ Soo Teik Lee $\cdot$ Dae Ghon Kim
}

Received: 9 May 2012/Accepted: 7 September 2012/Published online: 5 October 2012

(c) The International Gastric Cancer Association and The Japanese Gastric Cancer Association 2012

\begin{abstract}
Background When patients with advanced gastric cancer experience active bleeding, gastroenterologists normally choose between two treatment modalities, endoscopic hemostasis and transarterial embolization (TAE). In patients with advanced gastric cancer with bleeding, the predictive factors for endoscopic hemostatic failure are still unknown. Thus, the purpose of this study was to evaluate predictive factors for endoscopic hemostasis failure and to differentiate which hemostasis procedure is more effective for advanced gastric cancer with bleeding.

Methods We reviewed the medical records of patients who were diagnosed with advanced gastric cancer and acute non-variceal gastric bleeding from January 2006 to August 2011. Forty-five patients were enrolled in this study and they were divided into a group of 14 patients who had experienced successful endoscopic hemostasis and a group of 31 patients who had had unsuccessful hemostasis with the first endoscopy and then underwent TAE.

Results Lesion size and bleeding condition of Forrest class $1 \mathrm{a}$ or $1 \mathrm{~b}$ were statistically significant predictive factors for endoscopic hemostatic failure $(P=0.023$ and $P=0.017$, respectively). On multivariate logistic regression analysis, size (lesion $>2 \mathrm{~cm}$ ) was a significant predictive factor for endoscopic hemostatic failure [adjusted odds ratio (aOR) 8.056; $95 \%$ confidence interval (CI) 1.329-48.846].
\end{abstract}

K. H. Koh · K. Kim · D. H. Kwon · B. S. Chung ·

J. Y. Sohn - D. S. Ahn - B. J. Jeon - S. H. Kim ·

I. H. Kim · S. W. Kim ( $\)$ - S. O. Lee · S. T. Lee · D. G. Kim Department of Internal Medicine, Research Institute for Medical Science, Chonbuk National University College of Medicine, 634-18 Geumam-dong, Dukjin-ku, Jeonju,

Jeonbuk 561-712, Korea

e-mail: clickm@jbnu.ac.kr
Conclusions We determined that small bleeding lesions $(<2 \mathrm{~cm})$ and exposed vessels in the bleeding site with gastric cancer indicated that endoscopic hemostasis would be an effective hemostatic modality to choose. Particularly, in the opposite condition, the presence of large bleeding lesions $(>2 \mathrm{~cm})$ and non-exposed vessel bleeding with a tumor, endoscopic hemostasis failure is predicted and TAE could be recommended.

Keywords Bleeding - Endoscopic hemostasis · Gastric cancer · Transarterial embolization

\section{Introduction}

Active bleeding is one of the most serious and fatal complications in patients with advanced gastric cancer [1-5]. The incidence of gastric cancer with bleeding is reported to be about $1-8 \%$ [6-8]. For this reason, it is very important to establish a proper treatment plan for patients with gastric cancer accompanied by bleeding. Gastroenterologists usually choose between two treatment modalities for hemostasis, endoscopic hemostasis and transarterial embolization (TAE), depending on the patient's medical condition $[9,10]$. Because TAE is a more invasive procedure than endoscopic hemostasis, TAE is mostly performed after the failure of endoscopic hemostasis for gastric bleeding $[11,12]$. For this reason, endoscopic hemostasis is regarded as the first-line treatment and TAE is regarded as a first- or second-line treatment for gastric bleeding. However, in many cases, gastroenterologists encounter endoscopic hemostasis failure.

There have been several studies suggesting predictive factors for endoscopic hemostatic failure in patients with peptic ulcer bleeding, but such predictive factors have not 
been investigated in patients with gastric cancer bleeding $[13,14]$. Our study is the first to identify predictive factors for endoscopic hemostatic failure in patients with advanced gastric cancer with bleeding. Moreover, there have not been any comparative studies of the effects of endoscopic hemostasis and TAE in such patients. If we can establish predictive factors for endoscopic hemostatic failure, we can stabilize a patient's hemodynamics earlier, and this would improve their prognosis $[2,15]$. Thus, the purpose of this study was to identify factors predictive of endoscopic hemostasis failure and to determine which hemostatis procedure is more effective for patients with gastric cancer bleeding.

\section{Patients and methods}

\section{Patients}

We performed a single-center 5-year and 8-month (from January 2006 to August 2011) retrospective survey of all patients who were diagnosed with advanced gastric cancer and acute non-variceal gastric bleeding at Chonbuk National Hospital gastroenterology department. In total, 45 patients were enrolled retrospectively. The patients were divided into two groups; one group of 14 patients who had had successful endoscopic hemostasis (Group 1) and the other group of 31 patients who had had a failed endoscopic hemostasis prior to TAE (Group 2). For a comparative analysis, we collected the following data: prothrombin time (PT [INR]; cutoff value was 1.1), platelet (PLT) count, systolic blood pressure (SBP)/diastolic blood pressure (DBP), hemoglobin (hgb), number of transfused RBC units, number of users of nonsteroidal anti-inflammatory drugs (NSAIDs), bleeding site (lesser curvature, greater curvature, anterior wall, posterior wall), size of the bleeding site (cutoff value was $2 \mathrm{~cm}$ ), characteristics of the bleeding site vasculature (based on the Forrest classification, 1a or 1b), 30-day mortality, and arteries embolized during TAE (we were able to indirectly ascertain the information for the main problematic arteries when endoscopic hemostasis failed).

\section{Procedural technique}

Endoscopies were performed by one of eight skilled endoscopic specialists. If possible, we biopsied undiagnosed bleeding lesions that were considered to be gastric cancer based on endoscopic findings. The endoscopic specialists chose the hemostatic method, such as epinephrine injection, epinephrine spray, hemoclipping, or argon plasma coagulation, based on the patient's hemorrhagic site and clinical conditions. For TAE, after a common femoral artery puncture, a standard 5-Fr angiographic catheter was inserted through a 0.035 -inch guidewire to access the sites of suspected bleeding. Diagnostic pre-embolization arteriography was performed to identify and localize the bleeding site at the superior mesenteric artery or common hepatic artery, left gastric artery, and splenic artery via the celiac trunk. Then pre-embolization angiography was performed for the branched arteries such as the left gastroepiploic artery and the short gastric artery, right gastric artery, gastroduodenal artery, and right gastroepiploic artery. The extravasation of contrast or a pseudo-aneurysm pattern indicated active bleeding in the stomach, and abnormal vessels with mucosal blush were considered to indicate tumor staining. For patients who had active bleeding and tumor staining on pre-embolization arteriography, we tried to perform TAE using gelatin sponge particles, microcoil, and glue using a microcatheter with superselection of active bleeding vessels. If no bleeding was found on pre-embolization arteriography or no extravasation was seen despite the injection of contrast, embolization of the branches terminating at the clip, which was placed around the area of bleeding or the adjacent branches during pre-embolization endoscopy, was performed. After embolization, a final angiography was performed to confirm the success of the hemostasis. All angiographies and embolizations were performed by two interventional radiologists.

Successful endoscopic hemostasis was defined by the absence of bleeding seen in the endoscopic field, and the success of TAE was defined by the absence of extravasation of contrast seen on radiographic findings. The clinical success of the hemostasis was defined by the absence of hematemesis, melena, or hematochezia, in conjunction with stabilization of vital signs. Rebleeding was defined as the presence of hemorrhagic symptoms with reduced systolic blood pressure below $80 \mathrm{mmHg}$, or a greater than $2 \mathrm{~g} /$ $\mathrm{dl}$ decrease in hemoglobin in $24 \mathrm{~h}$ despite hydration and the transfusion of more than 2 units of packed red blood cells (PRBCs) to maintain a hemoglobin level of at least $10 \mathrm{~g} / \mathrm{dl}$.

\section{Statistical methods}

All variables in the study are presented as means and standard deviations (SD). Continuous variables were compared using the Student's $t$-test. Categorical data were analyzed using the $\chi^{2}$ test or Fisher's exact test, as appropriate. All $P$ values of $<0.05$ were considered statistically significant. All calculations were performed using SPSS statistical software version 18.0 (SPSS, Chicago, IL, USA). Odds ratios (ORs) and their associated $95 \%$ confidence intervals (95\% CIs) were obtained for all variables found to be significant by at least two multivariate analyses. 


\section{Results}

Characteristics of patients and gastric lesions

Technical success (bleeding target devascularization) was achieved in all patients, regardless of whether endoscopic hemostasis or TAE was performed. The mean patient (M: $\mathrm{F}=31: 14$ ) age was $67.58 \pm 15.10$ years, and the mean hemoglobin level was $8.22 \pm 2.23 \mathrm{~g} / \mathrm{dl}$. The 30 -day mortality rate of all the patients was $18.8 \%$, and all the patients who died were in Group 2 (endoscopic hemostasis failure group). No patients in Group 1 (endoscopic hemostasis success group) died within less than 30 days, and the 30-day mortality rate in Group 2 was $25.8 \%$. The most common tumor location was the posterior wall of the body, while a lesion in the lesser curvature was the second most common bleeding site. Out of all the patients, $86.7 \%$ had lesions larger than $2 \mathrm{~cm}$. There were 20 patients with gastric lesions of Forrest class 1a and 25 with gastric lesions of Forrest class 1b (Table 1).

Univariate analysis of outcomes of endoscopic hemostasis and TAE

The response to treatment and the standard for comparative analysis were determined based on whether or not there was rebleeding. Our comparative analysis revealed that there were no statistically significant differences between the two groups for PT level, PLT count, SBP, DBP, Hgb level, number of transfused $\mathrm{RBC}$ units, or number of

Table 1 Characteristics of patients and gastric lesions

\begin{tabular}{ll}
\hline Characteristics & $\begin{array}{l}\text { Patients and gastric } \\
\text { lesions }(N=45)\end{array}$ \\
\hline Age (years, mean $\pm \mathrm{SD})$ & $67.58 \pm 15.10$ \\
Sex (M:F) & $31: 14$ \\
PT (INR) (mean $\pm \mathrm{SD})$ & $1.14 \pm 0.21$ \\
PLT $(\times 1,000 \mathrm{U} / \mathrm{ml}$, mean $\pm \mathrm{SD})$ & $180.42 \pm 104.89$ \\
$\mathrm{SBP}(\mathrm{mmHg}$, mean $\pm \mathrm{SD})$ & $104.67 \pm 24.83$ \\
DBP $(\mathrm{mmHg}$, mean $\pm \mathrm{SD})$ & $66.44 \pm 12.64$ \\
$\mathrm{Hgb}(\mathrm{g} / \mathrm{dL}$, mean $\pm \mathrm{SD})$ & $8.22 \pm 2.23$ \\
RBC transfusion $(\mathrm{U}$, mean $\pm \mathrm{SD})$ & $2.378 \pm 1.01$ \\
NSAID use, $n(\%)$ & $11(24)$ \\
Location of tumor $(\mathrm{GC} / \mathrm{LC} / \mathrm{AW} / \mathrm{PW}), n$ & $7 / 13 / 6 / 19$ \\
Lesion size $(>2$ cm), $n(\%)$ & $39(86.7)$ \\
Bleeding condition $($ Forrest class $1 \mathrm{a} / \mathrm{b}), n$ & $20 / 25$ \\
Survival (survival rate, $n \%)$ & $37(82.2)$
\end{tabular}

$P T$ prothrombin time, INR international normalized ratio, $P L T$ platelets, $S B P$ systolic blood pressure, $D B P$ diastolic blood pressure, $H g b$ hemoglobin, $R B C$ red blood cells, NSAID non-steroidal antiinflammatory drug, $G C$ greater curvature, $L C$ lesser curvature, $A W$ anterior wall, $P W$ posterior wall, $S D$ standard deviation
NSAID users. The difference in survival rate between the two groups was statistically significant ( $p=0.037)$. In this study, the most meaningful data were for lesion size and bleeding conditions. For lesion size, $64 \%$ (9 of 14 patients) of Group 1 patients and $93.5 \%$ (29 of 31 patients) of Group 2 patients had tumor lesions larger than $2 \mathrm{~cm}$. Forrest class $1 \mathrm{a}$ and Forrest class $1 \mathrm{~b}$ bleeding condition status was observed in 71.4 and $28.6 \%$ of the patients in Group 1, respectively, and in 32.3 and $67.7 \%$ of the patients in Group 2, respectively. The differences in lesion size and bleeding condition were also statistically significant ( $p=0.023$ and $p=0.017$, respectively) (Table 2).

Multivariate logistic regression analysis of predictive factors

On multivariate logistic regression analysis, size (lesion $>2 \mathrm{~cm}$ ) [odds ratio (OR) 8.056; $95 \%$ confidence interval (CI) 1.329-48.846] was a significant predictive factor for endoscopic hemostatic failure. However, bleeding condition [odds ratio (OR) 2.552; $95 \%$ CI, 0.280-23.274] was statistically significant in the univariate analysis, but not in the multivariate analysis (Table 3 ). The remaining factors examined were not predictive for endoscopic hemostatic failure.

\section{Main problematic arteries}

In addition, we were able to obtain information on the main problematic arteries when TAE was performed after endoscopic hemostasis had failed. The main problematic artery on TAE was the left gastric artery (LGA) (Table 4; Fig. 1). Embolization at the single LGA was performed in 16 cases and LGA was the main problematic artery in 19 out of the 31 patients who underwent TAE.

\section{Discussion}

For the treatment of gastric cancer bleeding, endoscopic hemostasis is accepted as the first-line treatment, and it has previously been proven in several studies to be an effective treatment for the hemostasis of gastric bleeding $[5,6,8$, 16]. Even though endoscopic hemostasis is an effective method for gastric cancer bleeding, if an endoscopic approach for the bleeding lesion is difficult, or if the lesion has diffuse and massive bleeding, or if it has invaded a large main gastric vessel, it can be difficult to perform endoscopic hemostasis [7, 13, 14, 17, 18]. In this study, we demonstrated that TAE could be an alternative hemostasis treatment [19, 20]. TAE may be a better option than surgical treatment for gastric cancer bleeding with endoscopic hemostatic failure or rebleeding after endoscopic 
Table 2 Univariate analysis of outcomes of endoscopic hemostasis and transarterial embolization (TAE)

\begin{tabular}{|c|c|c|c|}
\hline & $\begin{array}{l}\text { Group } 1 \\
\text { Endoscopic hemostasis } \\
\text { success group }(n=14)\end{array}$ & $\begin{array}{l}\text { Group } 2 \\
\text { Endoscopic hemostasis } \\
\text { failure group }(n=31)\end{array}$ & $P$ value \\
\hline Age (years, mean $\pm \mathrm{SD}$ ) & $68.07 \pm 11.69$ & $67.35 \pm 16.59$ & 0.206 \\
\hline Sex $(M: F)$ & $10: 4$ & $21: 10$ & 0.547 \\
\hline $\mathrm{PT}(\mathrm{INR})($ mean $\pm \mathrm{SD})$ & $0.57 \pm 0.51$ & $0.52 \pm 0.51$ & 0.493 \\
\hline PLT $(\times 1,000 \mathrm{U} / \mathrm{ml}$, mean $\pm \mathrm{SD})$ & $244.71 \pm 129.82$ & $151.39 \pm 77.68$ & 0.357 \\
\hline $\mathrm{SBP}(\mathrm{mmHg}$, mean $\pm \mathrm{SD})$ & $110.00 \pm 29.08$ & $102.26 \pm 22.76$ & 0.650 \\
\hline $\mathrm{DBP}(\mathrm{mmHg}$, mean $\pm \mathrm{SD})$ & $68.57 \pm 12.92$ & $65.48 \pm 12.61$ & 0.572 \\
\hline $\mathrm{Hgb}(\mathrm{g} / \mathrm{dL}$, mean $\pm \mathrm{SD})$ & $9.08 \pm 1.87$ & $7.83 \pm 2.29$ & 0.267 \\
\hline $\mathrm{RBC}$ transfusion $(\mathrm{U}$, mean $\pm \mathrm{SD})$ & $2.29 \pm 0.99$ & $2.42 \pm 1.03$ & 0.976 \\
\hline NSAID use, $n(\%)$ & $6(42.9)$ & $5(16.1)$ & 0.062 \\
\hline Location of tumor (GC/LC/AW/PW), $n$ & $(0,4,3,7)$ & $(7,9,3,12)$ & 0.217 \\
\hline Lesion size $(>2 \mathrm{~cm}), n(\%)$ & $9(64)$ & $29(93.5)$ & $0.023 *$ \\
\hline Bleeding condition (Forrest class $1 \mathrm{a} / \mathrm{b}$ ), $n$ & $(10,4)$ & $(10,21)$ & $0.017 *$ \\
\hline Survival rate, $n(\%)$ & $14(100)$ & $23(74.2)$ & $0.037 *$ \\
\hline
\end{tabular}

Data in the two groups were compared using univariate linear regression analysis

* $P<0.05$, indicates significant difference

Table 3 Multivariate logistic regression analysis of predictive factors for endoscopic hemostasis failure

\begin{tabular}{llll}
\hline & $P$ value & $\begin{array}{l}\text { Odds } \\
\text { ratio }\end{array}$ & $95 \% \mathrm{CI}$ \\
\hline PT $($ INR $)$ & 0.555 & 0.533 & $0.66-4.299$ \\
PLT $(\times 1,000 \mathrm{U} / \mathrm{ml})$ & 0.059 & 0.987 & $0.973-1.001$ \\
$\mathrm{Hgb}(\mathrm{g} / \mathrm{dL})$ & 0.072 & 0.634 & $0.385-1.042$ \\
NSAID use & 0.333 & 0.304 & $0.027-3.387$ \\
Lesion size $(>2 \mathrm{~cm})$ & $0.023^{*}$ & 8.056 & $1.329-48.846$ \\
Bleeding condition & 0.406 & 2.552 & $0.280-23.274$ \\
$\quad$ Forrest class $1 \mathrm{a} / \mathrm{b})$ & & & \\
\hline
\end{tabular}

Data were analyzed using multivariate logistic regression analysis $C I$ confidence interval

* $P<0.05$, indicates significant correlation

Table 4 Vessel selected for embolization in TAE patients

\begin{tabular}{ll}
\hline Selected vessel & $\begin{array}{l}\text { Group 2; Endoscopic } \\
\text { hemostasis failure group } \\
(n=31)\end{array}$ \\
\hline Left gastric artery (LGA) & 16 \\
Right gastric artery (RGA) & 6 \\
LGA + RGA & 3 \\
$\begin{array}{l}\text { More than two arteries excluding } \\
\text { LGA and RGA }\end{array}$ & 6 \\
\hline
\end{tabular}

hemostasis if the expert interventional radiologists $\mathrm{s}$ are able to perform a TAE, particularly for patients who have advanced gastric cancer and cannot be treated with surgical resection $[2,15]$. The effectiveness of TAE, without significant complications, in patients with gastric bleeding has been noted in several studies [18-25]. However, delayed TAE after endoscopic hemostasis failure or any other hemostatic procedures can also have a worse prognosis than earlier TAE performed only after diagnostic endoscopy $[12,26]$. This could explain the differences in survival rate in the present study between the endoscopic hemostatic group and the TAE group.

Both treatments should be performed as early as possible. Therefore, the gastroenterologist must decide whether endoscopic hemostasis will be successful. The predictive factors for endoscopic hemostatic failure in peptic ulcer bleeding, but not in gastric cancer bleeding, have been shown in several studies, and they are: (1) hemodynamic instability, (2) active bleeding, (3) large ulcer size ( $>2 \mathrm{~cm})$, and (4) ulcer in the lesser gastric curve [13, 14]. We assumed that the predictive factors for endoscopic hemostatic failure in gastric cancer bleeding would be similar to those of peptic ulcer bleeding. In our Group 1 (endoscopic hemostasis success group), 5 of the 14 patients had a smaller lesion $(<2 \mathrm{~cm})$, and 9 of the 14 patients had a larger lesion $(>2 \mathrm{~cm})$. Also, all the patients in Group 1 survived after successful endoscopic hemostasis. If we judged from this above result, the relationship between the success rate of endoscopic hemostasis and the size of the bleeding lesion was unclear. However, in Group 2, 29 of the 31 patients with endoscopic hemostasis failure had a larger lesion $(>2 \mathrm{~cm})$. So finally, the factor of a larger lesion size $(>2 \mathrm{~cm})$ demonstrated a significant correlation with endoscopic hemostasis failure. Eventually, bleeding cancer size and bleeding condition of Forrest class 1a or $1 \mathrm{~b}$, which is thought to be similar to active bleeding, were found to be predictive factors for endoscopic hemostatic 

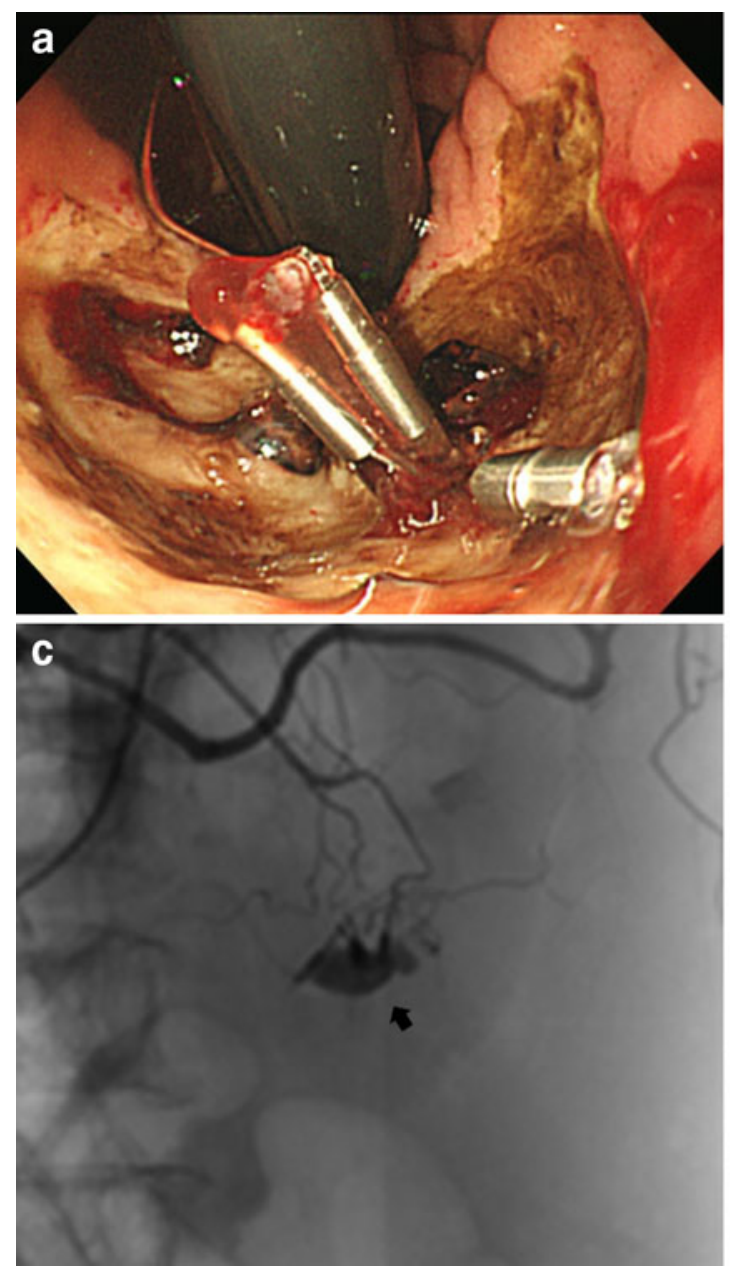

Fig. 1 Images in an 82-year-old woman with gastric cancer bleeding who experienced endoscopic hemostasis failure and who had subsequent transarterial embolization. a Gastric cancer with ulcer bleeding at the lesser curvature angle on endoscopy. Hemoclipping was performed to achieve hemostasis, but rebleeding occurred within $24 \mathrm{~h}$. b, c Selective angiography of the celiac trunk and superselective

failure in patients with gastric cancer bleeding in the present study [27]. In other words, in the endoscopic field, if the bleeding tumor size is more than $2 \mathrm{~cm}$ or the tumor exhibits active bleeding, such as Forrest class $1 \mathrm{~b}$, endoscopic hemostasis might fail stochastically. Additionally, we found that the main problematic artery in TAE was primarily the left gastric artery (LGA) (19 of the 31 cases), and this artery supplies the lesser curvature of the stomach, so TAE rather than endoscopic hemostasis should be considered if the gastric bleeding is seen in the LGA territory, such as in the lesser curvature of the stomach, on endoscopic findings. The most common location of the bleeding in the present study was the posterior wall of the stomach (19 out of 45 cases). This result is also consistent with the predictive factors identified for endoscopic hemostatic failure in peptic ulcer bleeding. It is possible that the reason that there is a difference in the locations
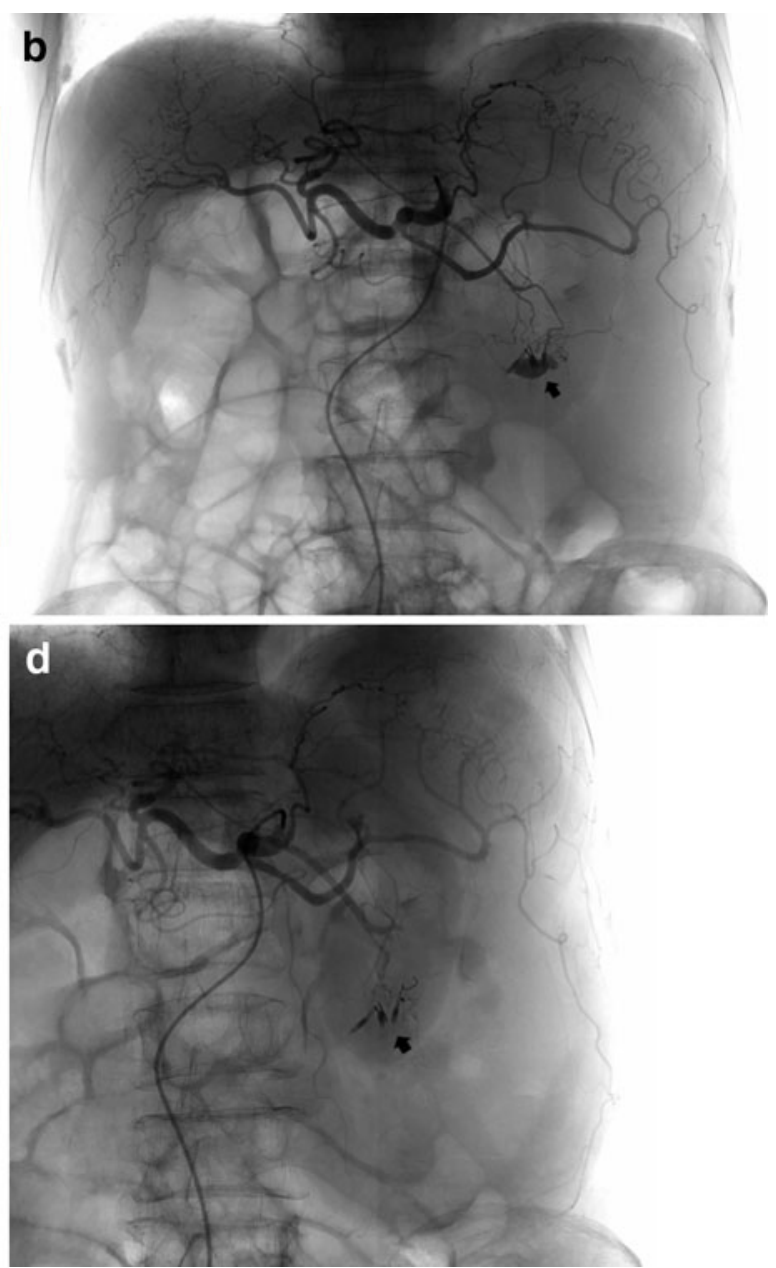

arteriogram of the left gastric artery, respectively, before embolization show extravasation of contrast medium. Three hemoclips were also seen around the bleeding focus (arrows). d Result after coil and glue embolization of the left gastric artery (arrow). Transarterial embolization was successful and the bleeding was controlled

between this study and former studies is that the territory with blood supply is not clear or the location makes it difficult to conduct hemostasis via an endoscopic technique. Finally, despite the lack of a statistically significant relationship between failure of endoscopic hemostasis and bleeding location, endoscopic hemostasis is also difficult in the lesser curvature of the stomach, which is supplied by the LGA.

In our study 8 of 41 patients died within 30 days after hemostasis with endoscopy and TAE. The 30-day mortality rate of all patients was $18.8 \%$ and all the patients who died were in Group 2. Three of the 8 patients who died within 30 days died of massive rebleeding after successful TAE. Four patients died owing to cancer progression, and 1 died of pneumonia. Given this cause of death, we can consider that this death was not directly related to the procedures of endoscopic hemostasis or TAE. 
There have not been any comparative studies of the treatment effectiveness of endoscopic hemostasis and TAE in patients with advanced gastric cancer with bleeding. In particular, to date, there have not been any studies dealing with predictive factors for endoscopic hemostatic failure in the endoscopic field, which would be helpful for determining the best treatment modality earlier in patients with advanced gastric cancer with bleeding. Thus, the results of the present study can help gastroenterologists decide, without hesitation, whether to perform endoscopic hemostasis or whether to perform an earlier TAE in order to improve the prognosis for gastric cancer patients with bleeding.

There are some limitations of this study. First, we authors deal with these two modalities, endoscopic hemostasis and hemostasis with TAE for the control of gastric cancer bleeding as a priority before we consider operating. In the present study, 5 of the 31 patients in Group 2 (endoscopic hemostasis failure group) had rebleeding at an average of 15 days after the procedure, and only 3 patients died from excessive rebleeding. All things considered, we can suggest that hemostasis with TAE was successful. Therefore, because we considered that hemostasis with endoscopy or TAE was sufficient to control the active bleeding of gastric cancer, we did not allow for gastric operations. Additionally, if gastric surgery could be indicated for the treatment of gastric cancer, surgery would be considered as soon as possible. However, most patients with gastric cancer bleeding visit the hospital via the emergency room and progress to an unstable vital condition. Thus, while we find the bleeding focus on the stomach with endoscopy, simultaneously we attempt hemostasis with endoscopy or TAE. Second, we did not gather a large amount of data from patients who did not undergo surgery. Almost all patients diagnosed with gastric cancer underwent surgery in advance, so the number of gastric cancer patients with bleeding that did not undergo surgery was small. Third, this study was a retrospective analysis without randomization of the patients. Fourth, there may have been a bias, in that differences in the patients' conditions, such as bleeding tendency and hemodynamic instabilities, between the two groups may have affected the survival rates of the two groups. However, in spite of these limitations, we performed a thorough review of the effectiveness of two hemostatic methods, endoscopic hemostasis and TAE, in patients with gastric cancer bleeding, and we also identified clinical factors that were associated with successful hemostatic treatment.

In conclusion, considering the endoscopic findings of the patients with advanced gastric cancer bleeding, a nonexposed vessel in the bleeding site and large bleeding tumor size $(>2 \mathrm{~cm})$ were significant predictive factors for endoscopic hemostasis failure. Therefore, when endoscopic hemostasis failure is expected, immediate change of procedure to performing a TAE would be effective to raise the hemostatic success rate. Ultimately, the predictive factors for endoscopic hemostatic failure in gastric cancer bleeding are very similar to the predictive factors for endoscopic hemostatic failure in peptic ulcer bleeding; that is, active bleeding, large ulcer size, and the presence of a lesser curvature ulcer. The results of this study could allow gastroenterologists to choose the best hemostatic modality immediately, and this would improve the patient's prognosis. To precisely determine the predictive factors for endoscopic hemostasis failure, further large-scale randomized studies are warranted.

Acknowledgments This study was supported by funds from the Chonbuk National University Hospital Research Institute of Clinical Medicine.

\section{References}

1. Shah MA, Kelsen DP. Gastric cancer: a primer on the epidemiology and biology of the disease and an overview of the medical management of advanced disease. J Natl Compr Cancer Netw. 2010;8(4):437-47.

2. Yusupbekov A, Djuraev M, Nosirov A, Egamberdiev D. Direct results of surgical treatment of locally advanced gastric cancer complicated by bleeding. Ann Oncol. 2009;20:49.

3. Asakura H, Hashimoto T, Harada H, Mizumoto M, Furutani K, Hasuike $\mathrm{N}$, et al. Palliative radiotherapy for bleeding from advanced gastric cancer: is a schedule of $30 \mathrm{~Gy}$ in 10 fractions adequate? J Cancer Res Clin. 2011;137(1):125-30. doi:10.1007/ s00432-010-0866-z.

4. Kim N. Screening and diagnosis of early gastric cancer. J Korean Med Assoc. 2010;53(4):290-8.

5. Akhtar K, Byrne JP, Bancewicz J, Attwood SEA. Argon beam plasma coagulation in the management of cancers of the esophagus and stomach. Surg Endosc-Ultras. 2000;14(12):1127-30.

6. Savides TJ, Jensen DM, Cohen J, Randall GM, Kovacs TOG, Pelayo E, et al. Severe upper gastrointestinal tumor bleeding: endoscopic findings, treatment, and outcome. Endoscopy. 1996; 28(2):244-8.

7. Webb WA, Mcdaniel L, Johnson RC, Haynes CD. Endoscopic evaluation of 125 cases of upper gastrointestinal-bleeding. Ann Surg. 1981;193(5):624-7.

8. Loftus EV, Alexander GL, Ahlquist DA, Balm RK. Endoscopic treatment of major bleeding from advanced gastroduodenal malignant lesions. Mayo Clin Proc. 1994;69(8):736-40.

9. Wong TCL, Wong KT, Chiu PWY, Teoh AYB, Yu SCH, Au $\mathrm{KWL}$, et al. A comparison of angiographic embolization with surgery after failed endoscopic hemostasis to bleeding peptic ulcers. Gastrointest Endosc. 2011;73(5):900-8. doi:10.1016/j.gie. 2010.11.024.

10. Pereira J, Phan T. Management of bleeding in patients with advanced cancer. Oncologist. 2004;9(5):561-70.

11. Defreyne L, Vanlangenhove P, De Vos M, Pattyn P, Van Maele G, Decruyenaere J, et al. Embolization as a first approach with endoscopically unmanageable acute nonvariceal gastrointestinal hemorrhage. Radiology. 2001;218(3):739-48.

12. Lee HJ, Shin JH, Shin JH, Yoon HK, Ko GY, Gwon DI, Song HY, et al. Transcatheter arterial embolization in gastric cancer patients with acute bleeding. Eur Radiol. 2009;19(4):960-5. doi: 10.1007/s00330-008-1216-2. 
13. Loffroy R. Bleeding peptic ulcers resistant to endoscopic treatment: calling for a surgeon or an interventional radiologist? Presse Med. 2011;40(2):123-5. doi:10.1016/j.lpm.2010.09.011.

14. Garcia-Iglesias P, Villoria A, Suarez D, Brullet E, Gallach M, Feu F, et al. Meta-analysis: predictors of rebleeding after endoscopic treatment for bleeding peptic ulcer. Aliment Pharmacol Ther. 2011;34(8):888-900. doi:10.1111/j.1365-2036.2011.04830.x.

15. Barkun AN, Bardou M, Kuipers EJ, Sung J, Hunt RH, Martel M, et al. International consensus recommendations on the management of patients with nonvariceal upper gastrointestinal bleeding. Ann Intern Med. 2010;152(2):101-13.

16. Seo HE, Jung YJ, Jung JK, Park CG, Kim DJ. Comparison of hemostatic efficacy between epinephrine injection alone and combined therapy with hemoclip for bleeding peptic ulcers. J Gastroenterol Hepatol. 2008;23:A127-A.

17. Jae HJ, Chung JW, Jung AY, Lee W, Park JH, et al. Transcatheter arterial embolization of nonvariceal upper gastrointestinal bleeding with N-butyl cyanoacrylate. Korean J Radiol. 2007;8(1): 48-56.

18. Loffroy R, Guiu B, D'Athis P, Mezzetta L, Gagnaire A, Jouve JL, et al. Arterial embolotherapy for endoscopically unmanageable acute gastroduodenal hemorrhage: predictors of early rebleeding. Clin Gastroenterol Hepatol. 2009;7(5):515-23. doi:10.1016/j.cgh. 2009.02.003.

19. Aina R, Oliva VL, Therasse E, Perreault P, Bui BT, Dufresne MP, et al. Arterial embolotherapy for upper gastrointestinal hemorrhage: outcome assessment. J Vasc Interv Radiol. 2001; 12(2):195-200.

20. Toyoda H, Nakano S, Takeda I, Kumada T, Sugiyama K, Osada $\mathrm{T}$, et al. Transcatheter arterial embolization for massive bleeding from duodenal ulcers not controlled by endoscopic hemostasis. Endoscopy. 1995;27(4):304-7. doi:10.1055/s-2007-1005697.

21. De Wispelaere JF, De Ronde T, Trigaux JP, de Canniere L, De Geeter T. Duodenal ulcer hemorrhage treated by embolization: results in 28 patients. Acta Gastroenterol Belg. 2002;65(1):6-11.

22. Dempsey DT, Burke, Reilly RS, McLean GK, Rosato EF. Angiography in poor-risk patients with massive nonvariceal upper gastrointestinal bleeding. Am J Surg. 1990;159(3):282-6.

23. Loffroy R, Rao P, Ota S, De Lin M, Kwak BK, Geschwind JF. Embolization of acute nonvariceal upper gastrointestinal hemorrhage resistant to endoscopic treatment: results and predictors of recurrent bleeding. Cardiovasc Interv Radiol. 2010;33(6):1088-100. doi:10.1007/s00270-010-9829-7.

24. Prochaska JM, Flye MW, Johnsrude IS. Left gastric artery embolization for control of gastric bleeding: a complication. Radiology. 1973;107(3):521-2.

25. Poultsides GA, Kim CJ, Orlando R 3rd, Peros G, Hallisey MJ, Vignati PV. Angiographic embolization for gastroduodenal hemorrhage: safety, efficacy, and predictors of outcome. Arch Surg. 2008;143(5):457-61. doi:10.1001/archsurg.143.5.457.

26. Funaki B. Endovascular intervention for the treatment of acute arterial gastrointestinal hemorrhage. Gastroenterol Clin N Am. 2002;31(3):701-13.

27. Heldwein W, Schreiner J, Pedrazzoli J, Lehnert P. Is the Forrest classification a useful tool for planning endoscopic therapy of bleeding peptic ulcers? Endoscopy. 1989;21(6):258-62. doi: 10.1055/s-2007-1010729. 